\title{
Biotransformación del pelo residual de curtiembres
}

\author{
Biotransformation of the residual hair from the tannery industry
}

\section{Ruth Viviana Numpaque P. ${ }^{1}$; Silvio Edgar Viteri R. ${ }^{2}$}

1 Estudiante de Maestría en Ciencias Biológicas. Universidad Pedagógica y Tecnológica de Colombia. Tunja, Boyacá, Colombia. ruthvian01@yahoo.es.

2 Docente, I.A. Ph.D. Facultad de Ciencias Agropecuarias, Universidad Pedagógica y Tecnológica de Colombia. Tunja, Boyacá, Colombia, silvio.viteri@uptc.edu.co.

Citar: NUMPAQUE, R.; VITERI, S. 2016. Biotransformación del pelo residual de curtiembres. Rev. Cienc. Agr. 33(2): 95-105. doi:http://dx.doi.org/10.22267/rcia.163302.56.

Recibido: Marzo 08 de $2016 . \quad$ Aceptado: Agosto 05 de 2016.

\section{RESUMEN}

La industria de curtiembres genera una alta carga contaminante representada por residuos líquidos, sólidos y gaseosos. Entre los residuos sólidos, el pelo residual es vertido directamente al río Bogotá y/o en rellenos sanitarios, desencadenando serios conflictos de orden ambiental, social y económico. El objetivo de esta investigación fue evaluar la capacidad de los microorganismos del EM y Agroplux en la transformación del pelo residual en compost. Se evaluaron tres tratamientos con pelo residual y materiales orgánicos de plaza en diferentes porcentajes y dos controles, utilizando el diseño completamente al azar, con tres repeticiones. Durante el compostaje se determinaron las variables temperatura, formación de agregados, pH y conductividad eléctrica. El desarrollo de la temperatura ocurrió en ciclos que concordaron con los volteos y re-inoculaciones. En el tercer ciclo el mayor incremento de temperatura fue obtenido en los tres tratamientos, cuando ya se había agotado la mayor parte de los residuos orgánicos. La formación de agregados indicó que el proceso se llevó a efecto principalmente en el tratamiento con $20 \%$ de pelo residual más $80 \%$ de residuos orgánicos. Además de su color oscuro y la ausencia de olor y fragmentos, el compost producido mediante dicho tratamiento mostró la conductividad eléctrica más baja y un $\mathrm{pH}$ casi neutro. Se concluye que los microorganismos del EM y Agroplux tienen capacidad para transformar el pelo residual en compost y por lo tanto representan una alternativa viable para la continuación del funcionamiento de la industria de curtiembres en Villapinzón (Cundinamarca) y en otras regiones de Colombia.

Palabras clave: compostaje, contaminantes industriales, curtido. 


\begin{abstract}
The tannering industry generates effluent with high contaminant load represented in a considerable amount of liquid, solid and gaseous waste. Among the solid wastes, the residual hair is dumped directly to the Bogota River or in landfills, triggering serious environmental, social and economic conflicts. The objective of this research was to evaluate the capacity of the EM and Agroplux microorganisms to transform the residual animal hair into compost. Three treatments, with different percentages of residual hair and urban organic residues and two controls, were evaluated, using a completely randomized experimental design, with three replications. During the composting process the variables temperature, aggregate formation, electrical conductivity and $\mathrm{pH}$ were evaluated. The development of temperature displayed cycles which were in accordance with the over turnings and re-inoculations of the compost piles. In the third cycle the greater increment in temperature was obtained in the three treatments, when the majority of the organic residues were almost completely decomposed. The aggregate formation indicated that the composting process took place principally in the treatment with $20 \%$ of residual hair and $80 \%$ of solid organic residues. In addition to the dark color and the absence of odor and of fragments of the original materials, the compost produced by means of this treatment showed the lower electrical conductivity and a pH almost reached to be neutral. It is concluded that the EM and Agroplux microorganisms have capacity to transform the residual hair into compost, and in consequence, they represent a viable alternative which can be adopted by the tannery industry to continue operating in Villapinzón (Cundinamarca) and in other regions of the country.
\end{abstract}

Key words: composting, industrial pollutants, tannings.

\section{INTRODUCCIÓN}

La industria del cuero, uno de los oficios de mayor arraigo y tradición en varias regiones de Colombia, ha ocupado un destacado lugar en la economía nacional (Herrera et al., 2015). Particularmente, en el municipio de Villapinzón (Cundinamarca), el establecimiento del sector curtiembres se remonta hacia los años 1950 y en la actualidad se encuentran alrededor de 120 curtidores, ubicados al sur del municipio y extendidos en un área de $7 \mathrm{Km}$, a lo largo del río Bogotá (Osorio et al., 2011b). La mayoría de las fábricas son de tipo familiar y la comunidad cuenta con un nivel educativo promedio de primaria, vive de una economía de subsistencia, utilizando una tecnología obsoleta (Osorio et al., 2011a).

El proceso de curtido transforma las pieles de animales bovinos, ovinos y porcinos en cuero (CNPML, 2004). El procedimiento consiste en remover de la piel el pelo, los elementos no estructurales y la grasa, para obtener al final un material durable, resistente e inmune. Según Switch (2011) el proceso de curtido consta de cuatro etapas: 1) Preparación, 2) Ribera, 3) Curtido y 4) Acabado. La etapa de ribera, en la cual se obtiene el pelo y se remueve la capa interior de la piel, que contiene el mayor contenido de grasa, es catalogada como la más riesgosa desde el punto de vista de contaminación ambiental (Reyes et al., 2009).

Del peso total de las pieles que ingresa a la curtiembre el $60 \%$ es eliminado, ya sea en las aguas residuales o como residuos sólidos que incluyen grasas, tejidos biodegradables, pelo, fibras, cuero curtido en forma de pedazos, viruta y polvo (Corredor, 2006). El pelo residual, en algunos casos durante el proceso es removido mediante filtración (Guzmán y Lujan, 2010) pero en la mayoría es descargado directamente al río Bogotá o al relleno 
sanitario, generando una problemática ambiental de alto impacto negativo sobre la calidad del agua (Osorio et al., 2011a) y el suelo. Por lo anterior, la industria de curtiembres ha sido reconocida no solo a nivel nacional sino también internacional como altamente contaminante, en virtud que los procesos de degradación ambiental que produce son generalmente de carácter irreversible (Corredor, 2006).

Se prevé que si se tomara las medidas y precauciones necesarias, la alta carga contaminante de la industria de curtiembres podría ser contrarrestada (DAMA, 2004), ya que sobre el tratamiento que debe darse a los residuos líquidos y sólidos provenientes de esta industria se han realizado muchas pruebas y estudios que optimizan la calidad de la disposición final de los mismos (Corredor, 2006); como es el caso del pelo residual, que podría transformarse en compost. El compostaje de residuos sólidos orgánicos es llevado a cabo por una gran diversidad de microorganismos, bajo el control de factores que afectan tanto su crecimiento como su actividad. La efectividad del proceso depende en gran medida de las condiciones ambientales, el método utilizado, las materias primas empleadas y otros factores que implican vigilancia constante para que estén en un rango óptimo (Román et al., 2013).

Con el fin de acelerar dicho proceso se han utilizado inóculos preparados con varios microorganismos (Cariello et al., 2007; Tortarolo et al., 2008; Vásquez et al., 2010; Viteri et al., 2015) y hasta con activadores biológicos como vinazas (Castillo et al., 2015). Entre los microorganismos figuran los del EM (Efficient microorganisms) y del Agroplux (AP). Los microorganismos del EM se utiliza en la estabilización, transformación y recuperación de desechos sólidos y líquidos generados por la producción agropecuaria, la industria de procesamiento de alimentos, fábricas de papel, mataderos y otros (APROLAB, 2007; Arias, 2010), constituyéndose así en una alternativa biológica generadora de bioinsumos, en armonía con el manejo res- ponsable del entorno (Sztern y Pravia, 1999). Los microorganismos del Agroplux, además de recuperar la biota del suelo, aportan nutrientes y sustancias biológicamente activas, como antibióticos y promotores de crecimiento vegetal (giberelinas, citoquininas, ácido indolacético y sus derivados) que favorecen el reciclaje de minerales, el desarrollo de los cultivos y evitan focos de contaminación (Gil et al., 2006; Agroplus, 2009).

El objetivo de esta investigación, fue evaluar la capacidad de los microorganismos del EM y del Agroplux para transformar el pelo residual de la industria de curtiembres en compost, en asocio con los microorganismos que naturalmente se encuentran en los residuos de plaza de mercado. En general, se trata del desarrollo de una técnica biológica, que sea viable y de acuerdo con el DAMA (2004) conduzca efectivamente al cumplimiento de la normativa ambiental por parte de la industria de curtiembres, al mejoramiento de su imagen ante la comunidad y en consecuencia a la continuación de su funcionamiento como industria no solamente en Villapinzón (Cundinamarca) sino también en otras regiones de Colombia.

\section{MATERIALES Y MÉTODOS}

Ubicación. El estudio se realizó en la Institución Educativa Escuela Normal Superior María Auxiliadora del municipio de Villapinzón, en el departamento de Cundinamarca. Villapinzón esta ubicado en el altiplano cundiboyacense, pertenece a la región natural del bosque andino y a la subregión de la cuenca alta del río Bogotá. $\mathrm{Su}$ cabecera municipal se encuentra localizada a 0513'09”LN y 7336 00 “LO, 2715 msnm y una temperatura media de $13^{\circ} \mathrm{C}$.

Recolección de materiales. Los materiales utilizados fueron pelo residual del proceso de pelambre de la industria de curtiembres, suministrado por una de las empresas curtidoras de tipo artesanal ubicadas en la zona, residuos orgánicos de plaza de mercado, aportados por los comerciantes 
de la plaza municipal de Villapinzón EM ${ }^{\circledR}$ y Agroplux $^{\circledR}$ (cultivos de microorganismos) obtenidos en la Fundación de Asesorías para el Sector Rural (FUNDASES).

El EM es un cultivo mixto de microorganismos benéficos, sin manipulación genética, presentes en ecosistemas naturales (Arias, 2010). Incluye levaduras (Saccharomyces cerevisiae Hansen), bacterias ácidolácticas (Lactobacillus cassei) y bacterias fotosintéticas (Rhodopseudomonas palustris), que promueven un proceso de fermentación antioxidante benéfico, aceleran la descomposición de la materia orgánica y promueven el equilibrio de la flora microbiana. El Agroplux (AP) es un cultivo líquido de microorganismos preparado con diversas especies de bacterias, hongos, levaduras y actinomicetos nativos de Colombia, producido mediante fermentación, con el fin de devolver el componente biótico a los suelos. Los dos bioinsumos son distribuidos por FUNDASES en Bogotá.

Montaje y manejo de las composteras. Las composteras se conformaron con la mezcla de pelo residual y residuos de plaza de mercado, previamente cortados en un tamaño de 3 a $5 \mathrm{~cm}$, en los porcentajes estipulados en cada tratamiento. El tamaño de cada compostera fue de $100 \mathrm{~cm}^{3}$, con capacidad para $200 \mathrm{~kg}$. El ajuste de la humedad, aproximadamente entre 60 y 70\%, se realizó mediante la prueba del puño, la cual consiste en coger un puñado de compost y apretarlo en la mano, si la mano se humedece pero no escurre líquido entre los dedos, la humedad es la adecuada (Palmero, 2010). El volteo de cada compostera se realizó cada 15 días.

Definición y aplicación de los tratamientos. La evaluación de la efectividad de los microorganismos del EM y AP sobre la transformación del pelo residual de las curtiembres, se llevó a efecto, en mezcla con residuos de plaza de mercado en composteras elaboradas sobre piso de cemento bajo techo. Los tratamientos evaluados fueron: T1, pelo residual $(20 \%)+$ residuos de plaza $(80 \%)+$ EM y AP; T2, pelo residual (40\%) + residuos de plaza $(60 \%)+$ EM y AP; T3, pelo residual $(60 \%)+$ residuos de plaza (40\%) + EM y AP. Se incluyeron dos controles: Residuos de plaza 100\% + EM y AP y Residuos de plaza 100\%, sin inoculación. El diseño utilizado fue completamente al azar, con tres repeticiones.

Siguiendo las recomendaciones de FUNDASES, la inoculación de EM y AP, se realizó al montaje de las composteras y en cada volteo. Al montaje, en cada compostera se aplicaron $500 \mathrm{~mL}$ de EM diluidos en 9L de agua y $2 \mathrm{~L}$ de AP diluidos en $2 \mathrm{~L}$ de agua y en cada volteo $166 \mathrm{~mL}$ de EM diluidos en 1,5L de agua y $660 \mathrm{~mL}$ de AP en 660mL de agua.

Variables evaluadas. Las variables evaluadas durante el proceso de compostaje, fueron temperatura, formación de agregados, $\mathrm{pH}$ y conductividad eléctrica. Para la temperatura se tomó una lectura, en horas de la mañana cada tercer día, con un termómetro de lectura instantánea. Para la formación de agregados se coleccionaron muestras representativas de $100 \mathrm{~g}$ por pila. Las muestras fueron secadas bajo sombra y luego tamizadas en un agitador compuesto por una serie de siete tamices $(4,75 ; 3,35 ; 2,00 ; 1,00$; $0,50 ; 0,212$ y $0,038 \mathrm{~mm})$, la cantidad de agregados resultante en cada tamiz, se cuantificó por medio del peso en una balanza. La conductividad eléctrica se determinó por medio de un conductivímetro en relación compost: agua $1: 1$. El pH, también se midió con esta relación, siguiendo el protocolo descrito por el IGAC (2006). Al terminar el experimento, del tratamiento que mostró el mayor grado de descomposición y de cada control, se tomó una muestra del producto final y se llevó al laboratorio para el análisis de fertilidad.

Análisis estadístico. Los datos de las variables cantidad de agregados y conductividad eléctrica, fueron sometidos al Análisis de Varianza y con base en la significancia se realizó la comparación de medias mediante la prueba Tukey, utilizando el paquete estadístico SPSS 22 (IBM, 2013). 


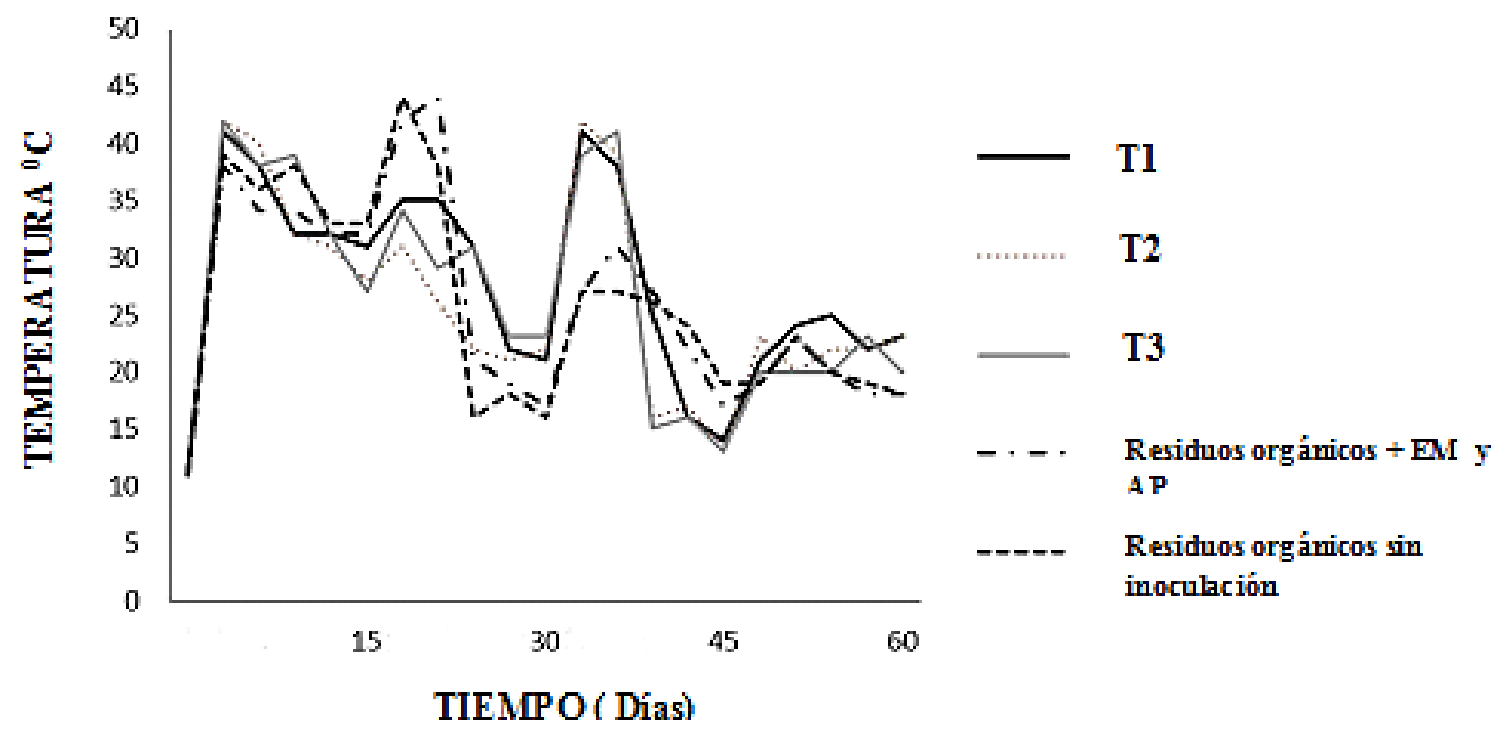

Figura 1. Desarrollo de la temperatura durante el proceso de compostaje.

\section{RESULTADOS Y DISCUSIÓN}

En concordancia con López (2010), la transformación ocurrida en las pilas de compostaje se llevó a efecto como resultado de la actividad microbiana ejercida sobre el pelo residual y los residuos orgánicos incluidos en el estudio, es así como Las observaciones periódicas realizadas en las composteras permitieron apreciar cambios físicos, como reducción de volumen, tamaño y olor, que fueron indicativos de la descomposición de los materiales utilizados, incluyendo el pelo residual. En la Figura 1 se puede observar que el desarrollo de la temperatura en las composteras se presentó en ciclos que concordaron con los volteos y las re-inoculaciones. Al tercer día de haberse iniciado el proceso, la temperatura en los tratamientos y controles subió hasta un rango entre 42 y $44^{\circ} \mathrm{C}$ y luego descendió hasta el rango entre 27 y $38^{\circ} \mathrm{C}$, en el día 15 . El incremento en temperatura en este primer ciclo evidenció el inicio de la descomposición de los materiales por los microorganismos, utilizando según Román et al. (2013) fuentes sencillas de C y N y generando calor.

En el segundo ciclo, después del primer volteo y la primera re-inoculación, la temperatura mostró un nuevo ascenso, acentuado en los controles y moderado en los tratamientos. En los controles, al no incluir pelo residual, el proceso de degradación continuó de acuerdo con lo indicado por Cuervo (2010). El ascenso, en general, fue seguido de un rápido descenso hasta el rango entre 17 y $23^{\circ} \mathrm{C}$, notorio especialmente en los controles.

En contraste, en el tercer ciclo, después del respectivo volteo y re-inoculación, el ascenso en temperatura ocurrió en los tratamientos y no en los controles, similar a lo reportado por Bello et al. (2011), quienes al incorporar pelo residual en las pilas de compostaje, registraron un aumento en la temperatura, en las semanas cinco y siete. En los tratamientos, el ascenso fue hasta 39 y $43^{\circ} \mathrm{C}$ y en los controles hasta 27 y $31^{\circ} \mathrm{C}$. Esta observación permite inferir que al tiempo que se agotaba gradualmente la cantidad de residuos orgánicos en los tratamientos, la degradación del pelo residual permitió la continuación del proceso de compostaje.

En el cuarto ciclo, después del tercer volteo y la respectiva re-inoculación, se presentó un moderado ascenso de la temperatura tanto en los tratamientos como en los controles, destacándose el tratamiento T1, que contenía el $20 \%$ de pelo residual y $80 \%$ de residuos de plaza de mercado, co- 
incidiendo con Cuervo (2010), quien en composteras con pelo residual obtuvo también un rápido ascenso en los perfiles de temperatura en el día 45 del proceso, como resultado de la sucesiva transformación de la materia orgánica por parte de la carga microbiana introducida. Después del cuarto volteo, a los 60 días, la temperatura se mantuvo en el rango entre 15 y $25^{\circ} \mathrm{C}$, con cambios leves entre tratamientos y controles.

Aunque el desarrollo de la temperatura ocurrió en ciclos como resultado de los volteos y re-inoculaciones, es preciso anotar, que en ninguno de ellos alcanzó $66^{\circ} \mathrm{C}$ como lo indican otros autores, entre ellos Cariello et al. (2007), en la transformación de lodos residuales con microorganismos endógenos, Nair y Okamitsu (2010) en el compostaje de residuos de cocina mediante inoculantes microbianos y Viteri et al. (2015) en la transformación de residuos de plaza de mercado con bacterias celulolíticas y Castillo et al. (2015), con hongos celulolíticos. El factor más limitante, fue la temperatura del sitio donde se llevó a efecto el estudio, la cual fluctúa entre 0 y $5^{\circ} \mathrm{C}$, principalmente en la media noche y en la madrugada.

Sin embargo, la curva de temperatura, reflejó una respuesta positiva a los volteos y re-inoculaciones y ello es prueba que el proceso de descomposición de los residuos utilizados si se efectúo, conduciendo a su transformación en compost.
Bajo las condiciones de temperatura del sitio donde se desarrolló el estudio, según Sylvia et al. (2005), el proceso se llevó a efecto, principalmente en la fase mesofílica, cuyo rango óptimo de temperatura para el crecimiento y actividad de los microorganismos oscila entre 15 y $40^{\circ} \mathrm{C}$. En la fase termofílica la temperatura avanzó únicamente hasta $43^{\circ} \mathrm{C}$, sin dar opción a la segunda sucesión de microorganismos termofílicos que generalmente ocurre alrededor de los $60^{\circ} \mathrm{C}$.

Cantidad de agregados. En concordancia con los ciclos de temperatura (Figura 1), la transformación de los materiales en agregados, progresó gradualmente durante el proceso en cada una de las composteras, con diferencias significativas entre tratamientos y entre controles (Tabla 1). La formación de agregados en los controles resultó significativamente superior a los tratamientos en cada uno de los muestreos, con diferencias significativas a favor del control sin inoculación, a partir del día 90 hasta el fin del proceso. Los resultados obtenidos en los controles contrastan con los reportados por Viteri et al. (2015) y Castillo et al. (2015), quienes encontraron, que la inoculación de los microorganismos del EM y del Agroplux en el compostaje de residuos de plaza de mercado y de flores no produjo efectos que se diferencien de los producidos por los microorganismos celulolíticos específicos en asociación con los nativos.

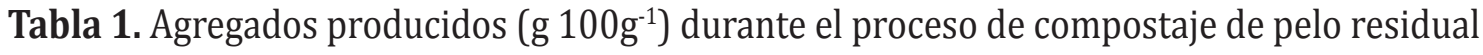
de la industria de curtiembres con residuos orgánicos, inoculados con EM y AP.

\begin{tabular}{lcrrrrrrrr}
\hline \multirow{2}{*}{ Tratamiento } & \multicolumn{10}{c}{ Días del muestreo } \\
\cline { 2 - 10 } & $\mathbf{3 0}$ & $\mathbf{4 5}$ & $\mathbf{6 0}$ & $\mathbf{7 5}$ & $\mathbf{9 0}$ & $\mathbf{1 2 0}$ & $\mathbf{1 5 0}$ & $\mathbf{1 6 5}$ & $\mathbf{1 8 0}$ \\
\hline T1 & $1,7^{\mathrm{a}}$ & $2,2^{\mathrm{a}}$ & $2,2^{\mathrm{a}, \mathrm{b}}$ & $4,1^{\mathrm{b}}$ & $6,1^{\mathrm{c}}$ & $5,2^{\mathrm{b}}$ & $10,2^{\mathrm{b}}$ & $13,2^{\mathrm{b}}$ & $21,8^{\mathrm{c}}$ \\
T2 & $2,5^{\mathrm{a}}$ & $2,0^{\mathrm{a}}$ & $3,2^{\mathrm{b}}$ & $4,2^{\mathrm{b}}$ & $4,4^{\mathrm{b}}$ & $4,9^{\mathrm{b}}$ & $5,1^{\mathrm{a}}$ & $5,1^{\mathrm{a}}$ & $3,1^{\mathrm{a}}$ \\
T3 & $1,5^{\mathrm{a}}$ & $1,7^{\mathrm{a}}$ & $2,0^{\mathrm{a}}$ & $2,5^{\mathrm{a}}$ & $2,8^{\mathrm{a}}$ & $3,4^{\mathrm{a}}$ & $4,4^{\mathrm{a}}$ & $6,2^{\mathrm{a}}$ & $7,6^{\mathrm{b}}$ \\
Residuos + EM y AP & $11,9^{\mathrm{b}}$ & $36,2^{\mathrm{c}}$ & $21,5^{\mathrm{c}}$ & $25,7^{\mathrm{d}}$ & $30,0^{\mathrm{d}}$ & $31,6^{\mathrm{c}}$ & $38,4^{\mathrm{c}}$ & $33,0^{\mathrm{c}}$ & $41,6^{\mathrm{d}}$ \\
Residuos sin & $19,2^{\mathrm{b}}$ & $32,4^{\mathrm{b}}$ & $27,3^{\mathrm{d}}$ & $21,1^{\mathrm{c}}$ & $35,1^{\mathrm{e}}$ & $50,7^{\mathrm{d}}$ & $58,3^{\mathrm{d}}$ & $53,8^{\mathrm{d}}$ & $63,5^{\mathrm{e}}$ \\
Inoculación & & $*$ & $*$ & $*$ & $*$ & $*$ & $*$ & $*$ & $*$ \\
Significación & $*$ & $*$ & $*$ & $*$ & $*$ & $*$ & $*$ \\
\hline
\end{tabular}

Promedios con letras diferentes difieren significativamente, prueba Tukey $(\mathrm{P} \leq 0,05)$. 
Respecto a los tratamientos, las diferencias en cuanto a la formación de agregados, empezaron a ser significativas a partir del día 60, superando significativamente el tratamiento $\mathrm{T} 1$ a los tratamientos T2 y T3, a partir de los 150 días del proceso. A medida que aumentó la proporción de pelo residual en los tratamientos, disminuyó la formación de agregados, poniendo de manifiesto, que la concentración de sales en el pelo residual, es un factor para la actividad microbiana y por lo tanto, la proporción de dicho insumo con respecto a los residuos orgánicos, no debe ser superior al 20\%. La mayor cantidad de agregados, se formó en los tamaños comprendidos entre 4,75 y $1 \mathrm{~mm}$.

Conductividad eléctrica (CE). La variación de la CE en los tratamientos utilizados en el estudio se muestra en la Tabla 2.

Lo primero que se puede resaltar, es una considerable diferencia entre los valores correspondientes a los tratamientos con pelo residual $(10,6$ a $21,2 \mathrm{dS}$ $\left.\mathrm{m}^{-1}\right)$ y los controles $\left(6,7 \mathrm{a} 8,6 \mathrm{dSm}^{-1}\right)$. En los tratamientos, el valor de la CE, resultó en proporción directa a la cantidad de pelo residual, registrándose por lo tanto, los mayores valores en el T3. Tanto en los tratamientos como en los controles, la $\mathrm{CE}$, aumentó gradualmente durante el proceso de compostaje, lo cual indica que el contenido de sales se incrementó a medida que avanzó la descomposición de los materiales.

En cuanto a los resultados de los tratamientos con pelo residual, Barrena et al. (2007), al trabajar en compostaje de residuos de pelo de la industria del curtido con destintado y lodos de aguas residuales municipales, encontró que la CE también aumentó durante el proceso, probablemente debido al incremento en la concentración de nutrientes, tales como nitratos durante la transformación de compuestos orgánicos (Sánchez et al. 2001). En cuanto a los controles, los valores resultaron superiores a los reportados por Viteri et al. (2015) y dentro del rango indicado por Castillo et al. (2015). El aumento de la CE, como resultado de la concentración de sales en los insumos y del proceso de descomposición, constituye una alerta para el potencial de uso del producto final, ya que afecta considerablemente el crecimiento, sobre todo en especies sensibles (Costa et al., 2010). De acuerdo con Sánchez (2001) y Bueno et al. (2008), la CE de un compost, depende de la naturaleza y la composición del material aportado, fundamentalmente por su concentración de sales y en menor grado por la presencia de iones amonio o nitrato formados durante el proceso.

Tabla 2. Variación de la $\mathrm{CE}\left(\mathrm{dS} \mathrm{m}^{-1}\right)$ durante el proceso de compostaje de pelo residual de la industria de curtiembres con residuos orgánicos, inoculados con EM y AP.

\begin{tabular}{|c|c|c|c|c|c|c|c|c|c|}
\hline \multirow{2}{*}{ Tratamiento } & \multicolumn{9}{|c|}{ Días del muestreo } \\
\hline & 30 & 45 & 60 & 75 & 90 & 120 & 150 & 165 & 180 \\
\hline T1 & $10,6^{\mathrm{b}}$ & $9,1^{b}$ & $11,3^{b}$ & $14,9^{\mathrm{c}}$ & $12,1^{\mathrm{b}}$ & $13,8^{b}$ & $14,7^{c}$ & $13,4^{b}$ & $12,9^{b}$ \\
\hline $\mathrm{T} 2$ & $12,0^{\mathrm{b}}$ & $11,0^{\mathrm{b}}$ & $13,3^{c}$ & $14,6^{c}$ & $13,6^{c}$ & $13,9^{b}$ & $13,0^{b}$ & $14,5^{c}$ & $14,7^{\mathrm{c}}$ \\
\hline T3 & $18,0^{c}$ & $16,9^{\mathrm{c}}$ & $15,3^{d}$ & $19,3^{d}$ & $18,8^{d}$ & $19,8^{c}$ & $19,5^{\mathrm{d}}$ & $20.0^{\mathrm{d}}$ & $21.2^{\mathrm{d}}$ \\
\hline Residuos + EM y AP & $6,7^{\text {a }}$ & $5,4^{\mathrm{a}}$ & $7,1^{\text {a }}$ & $7,7^{\mathrm{b}}$ & $7,6^{\mathrm{a}}$ & $6,0^{\mathrm{a}}$ & $8,6^{\mathrm{a}}$ & $8,6^{\mathrm{a}}$ & $8,6^{\mathrm{a}}$ \\
\hline Residuos sin Inoculación & $6,7^{\mathrm{a}}$ & $9,2^{b}$ & $7,3^{\mathrm{a}}$ & $6,1^{\mathrm{a}}$ & $7,4^{\text {a }}$ & $6,2^{\mathrm{a}}$ & $7,6^{\mathrm{a}}$ & $8,2^{\mathrm{a}}$ & $8,4^{\mathrm{a}}$ \\
\hline significación & $*$ & $*$ & $*$ & $*$ & $*$ & $*$ & $*$ & $*$ & $*$ \\
\hline
\end{tabular}

Promedios con letras distintas difieren significativamente, prueba Tukey $(\mathrm{P} \leq 0,05)$. 
pH. La Tabla 3, muestra que durante el proceso, las fluctuaciones del $\mathrm{pH}$ en los materiales fueron leves en los tratamientos con pelo residual y un poco más marcadas en los controles. En los tratamientos, a diferencia de los tratamientos T2 y T3, en el T1 se presentó una leve tendencia hacia la acidificación, desde pH8.1 hasta pH7,1. Estos resultados concuerdan con Bello et al. (2011) quienes, mediante su investigación en el compostaje de pelo residual del proceso de pelambre, reportaron ligeros cambios de $\mathrm{pH} 8,2$ a pH 7,4. Contrario a lo anterior, en los controles con solo residuos de plaza, inicialmente se presentó una leve tendencia hacia la basicidad seguida de un ligero descenso hasta el final del proceso. Esta variación en los controles, concuerda con otros autores quienes indican que en la etapa termofílica del proceso de compostaje, el $\mathrm{pH}$ sube y alcaliniza el medio (Labrador, 2001; Román et al., 2013) y en la etapa final, tiende a la neutralidad, debido a la generación de compuestos húmicos que tienen un efecto tampón sobre los cambios de $\mathrm{pH}$ (Bueno et al, 2008). Según Sáez (2000), el pH ideal para compostaje está entre 6,5 y 8,0, pero la capacidad natural del proceso, hace posible un rango más amplio por lo cual el compostaje puede funcionar efectivamente entre un pH5,5 y 9,0.

Fertilidad del compost. A los 237 días del proceso, se seleccionó el compost obtenido en el T1. La selección se hizo con base en su color oscuro y ausencia de olor y de fragmentos del material original, características físicas que entre los otros dos tratamientos indicaron el mejor grado de descomposición (CONAMA, 2000). Adicionalmente, en comparación con los tratamientos T2 y T3, en dicho tratamiento se presentó la mejor temperatura en la etapa de estabilización (Figura 1), la mayor formación de agregados $\left(21,8 \mathrm{~g} 100 \mathrm{~g}^{-1}\right)$ (Tabla 1), la CE más baja $\left(12,9 \mathrm{dS} \mathrm{m}^{-1}\right.$ ) (Tabla 2) y un pH casi neutro $(7,1)$ (Tabla 3 ), que lo potencializa para ser aplicado en cualquier tipo de suelo (Bohórquez et al., 2014). Los resultados del análisis de fertilidad, en comparación a los controles, se consignan en la Tabla 4.

Tabla 3. Variación del pH durante el proceso de compostaje de pelo residual de la industria de curtiembres con residuos orgánicos, inoculados con EM y AP.

\begin{tabular}{lccccccccc}
\hline \multirow{2}{*}{ Tratamiento } & \multicolumn{7}{c}{ Días del muestreo } \\
\cline { 2 - 9 } & $\mathbf{3 0}$ & $\mathbf{4 5}$ & $\mathbf{6 0}$ & $\mathbf{7 5}$ & $\mathbf{9 0}$ & $\mathbf{1 2 0}$ & $\mathbf{1 5 0}$ & $\mathbf{1 6 5}$ & $\mathbf{1 8 0}$ \\
\hline T1 & 8,0 & 8,3 & 7,9 & 8,1 & 7,9 & 7,3 & 7,3 & 7,2 & 7,1 \\
T2 & 7,4 & 8,0 & 7,9 & 7,8 & 8,1 & 7,8 & 7,6 & 7,6 & 7,6 \\
T3 & 7,6 & 7,2 & 7,9 & 7,8 & 7,9 & 7,6 & 7,7 & 7,6 & 7,5 \\
Residuos + EM y AP & 6,7 & 8,0 & 8,1 & 8,0 & 8,2 & 8,2 & 8,1 & 8,1 & 8,0 \\
Residuos sin Inoculación & 6,7 & 7,3 & 8,2 & 7,9 & 7,5 & 7,4 & 7,4 & 7,3 & 7,3 \\
\hline
\end{tabular}

Tabla 4. Análisis de fertilidad del compost obtenido en el T1, en comparación a los controles.

\begin{tabular}{|c|c|c|c|c|c|c|c|c|}
\hline Tratamiento & $\mathrm{pH}$ & $\begin{array}{l}\text { M0 } \\
(\%)\end{array}$ & $\underset{(p p m)}{P}$ & $\underset{\text { (meq.100g }}{\mathrm{Ca}}$ & $\underset{\text { (meq.100g-1) }}{\mathrm{Mg}}$ & $\underset{\text { (meq.100g-1) }}{\mathrm{K}}$ & $\underset{\text { (meq.100g-1) }}{\mathrm{Na}}$ & $\begin{array}{c}\mathrm{CE} \\
\left.\text { (dS.m } \mathrm{m}^{-1}\right)\end{array}$ \\
\hline T1 & 7,2 & 30,1 & 497 & 38,4 & 7,3 & 15,7 & 27,6 & 16,6 \\
\hline Residuos + EM y AP & 7,6 & 28,7 & 740 & 22,0 & 13,5 & 49,9 & 6,0 & 9,2 \\
\hline Residuos sin Inoculación & 7,2 & 30,2 & 688 & 20,3 & 10,0 & 29,1 & 5,5 & 9,10 \\
\hline
\end{tabular}


El contenido de materia orgánica, mayor del 20\% y el pH 7,2 cumplen con los requerimientos establecidos por la Norma Chilena de Calidad de Compost, NCh2880 (INN, 2005). Según Rodríguez y Córdova (2006), el contenido de fósforo es bajo, puesto que el límite es de 1000ppm pero el de potasio, al superar los 6,39meq100g-1, es adecuado. El conte-

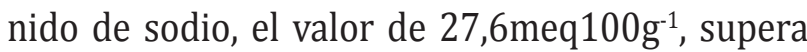
ampliamente el límite permisible establecido en la Norma de Calidad de compost (CONAMA, 2000), pero era esperado, puesto que en las operaciones de depilado se utiliza sulfuro de sodio (Bello et al., 2011). En cuanto al contenido de magnesio el valor no supera el límite máximo de $23 \mathrm{meq} 100 \mathrm{~g}$ ${ }^{1}$ permitido para biosólidos de excelente calidad (USEPA, 1995).

En relación a la $\mathrm{CE}$, el valor obtenido en el producto final es mayor a los de 9,9 y 12,1dS m ${ }^{-1}$ indicados por Bello et al. (2011) y a los valores comprendidos entre 5,43 y $8,29 \mathrm{dS} \mathrm{m}{ }^{-1}$ reportados por Barrena et al. (2007), en el compostaje de residuos de pelo de la industria del curtido con destintado y lodos de aguas residuales municipales, pero menor con respecto al resultado de $28,1 \mathrm{dS} \mathrm{m}^{-1}$ obtenido por Contreras et al. (2004), en el compostaje de los efluentes de curtiduría con estiércol de vaca y paja de trigo. Dado que dicho valor $\left(16,6 \mathrm{dS} \mathrm{m}^{-1}\right)$ sobrepasa ampliamente los límites establecidos por la Norma Chilena de Calidad de Compost, NCh2880 (INN, 2005), se prevé, que su uso debe ser incorporandolo al suelo, donde además de ayudar a neutralizar la acidez contribuiría a reducir los costos de encalamiento (Castillo et al., 2015). La dosis de compost que puede incorporarse depende de la $\mathrm{CE}$, puesto que un exceso de salinidad en la solución del suelo dificulta la absorción de agua por las raíces de las plantas, de modo que en algunos casos sólo prosperan las especies resistentes (Bueno et al., 2008).

En resumen, los resultados de esta investigación, muestran que los microorganismos del EM y Agroplux, en asocio con los microorganismos nativos, tienen potencial para transformar un residuo industrial de alto poder contaminante como lo es el pelo residual de la industria de curtiembres en compost, un producto que de ser aplicado apropiadamente de acuerdo con sus características, es de vital importancia para el sector agrícola. Al garantizar mediante la aplicación de esta técnica biológica, la reducción de los riesgos de contaminación ambiental, dicha industria podría justificar la continuación de su funcionamiento no solo en Villapinzón (Cundinamarca) sino también en otras regiones del país y del mundo en general.

\section{CONCLUSIONES}

La degradación del pelo residual en el proceso de compostaje se llevó a efecto después de los 30 días, cuando ya se había agotado gran parte de los residuos sólidos orgánicos.

El mejor grado de descomposición lo mostró el tratamiento con $20 \%$ de pelo residual más $80 \%$ de residuos de plaza de mercado y la inoculación de EM y Agroplux. Además de su color oscuro y la ausencia de olor y de fragmentos de los materiales inicialmente utilizados el compost producido por medio de dicho tratamiento, mostró el mayor porcentaje de formación de agregados, la conductividad eléctrica más baja y el pH más cercano a la neutralidad.

Los valores de conductividad eléctrica en el compost producido, indican que su uso se realice en mezcla con suelo o con otros sustratos, en proporciones no mayores del $20 \%$.

La biotransformación del pelo residual de la industria de curtiembres en compost representa una alternativa viable para disminuir los riesgos de contaminación ambiental y en consecuencia permitir la continuación del funcionamiento de dicha industria en el país y en el mundo en general. 


\section{REFERENCIAS BIBLIOGRÁFICAS}

AGROPLUS. 2009. El portal de los ganaderos. En: La ganadería.org. http://www.laganaderia.0rg/15/index.ph p?view=article \&catid=1\%Atimas\&id=109\%3Aagroplu s\&format=pdf\&option=com_content\&Itemid $=41$; consulta: enero, 2016.

APROLAB. PROGRAMA DE APOYO A LA FORMACIÓN PROFESIONAL PARA LA INSERCIÓN LABORAL. 2007. Manual para la producción de Compost con Microorganismos Eficaces. En: http:// www.em-la.com/archivosde usuario / base_datos / manual _ para_elaboracion_ de_compost.pdf; consulta: julio, 2013.

ARIAS, A. 2010. Microorganismos eficientes y su beneficio para la agricultura y el medio ambiente. J. de Ciencia e Ingeniería. 2(02):42 - 45.

BARRENA, R.; PAGANS, E.; ARTOLA, A.; VÁSQUEZ, F.; SÁNCHEZ, A. 2007. Co-composting of hair waste from the tanning industry with de-inking and municipal wastewater sludges. Biodegradation 18:257 - 268. Doi: 10.1007/s10532-006-9060-z.

BELLO, T.; OSORIO, L.; SANZ, M.; SANTOS, T. 2011. Description of the demo process for the composting of hair from the de-hairing process and fleshings from the liming process, including the results of the environmental and economic impact assessment and process of knowledge transfer. Primera edición. Opciones gráficas editores. Colombia. 23 p.

BOHÓRQUEZ, A.; PUENTES, Y.; MENJIVAR, J. 2014. Evaluación de la calidad del compost producido a partir de subproductos agroindustriales de caña de azúcar. Corpoica Ciencia y Tecnología Agropecuaria. 15(1):73 - 81

BUENO, P.; DÍAZ, M.; CABRERA, F. 2008. Factores que afectan al proceso de compostaje. En: http://digital. csic.es/bitstream/10261/20837/3/Factores; consulta: junio, 2016.

CARIELLO, M.; CASTAÑEDA, L.; RIOBO, I.; GONZÁLEZ, J. 2007. Endogenous microorganisms inoculant to speed up the composting process of urban sewage sludge. $R$. C. Suelo Nutr. Veg. 7(3):26 - 35.

CASTILLO, D.; VITERI, P.; VITERI, S. 2015. Desarrollo y evaluación de un inoculo de hongos celuloliticos. Rev. U.D.C.A Act. \& Div. Cient. 18(1):217 - 226.

CNPML. CENTRO NACIONAL DE PRODUCCIÓN MÁS LIMPIA Y TECNOLOGÍAS AMBIENTALES. 2004. Proyecto Gestión Ambiental en la Industria de Curtiembre en Colombia - Manual Ambiental Sectorial. En: http:// www.tecnologiaslimpias.org/curtiembres/ manual. pdf; consulta: febrero, 2016.

CONAMA. COMISIÓN NACIONAL DEL MEDIO AMBIENTE. 2000. NORMA DE CALIDAD DE COMPOST.. En: http:// www.lombricultura.cl/lombricultura.cl/userfiles/file/biblioteca/normas/Norma\%20 calidad \% 20 COMPOST.pdf; consulta: junio, 2016.

CONTRERAS, D.; ALVAREZ, N.; TRUJILLO; DENDOOVEN, L. 2004. Composting of tannery effluent with cow manure and wheat Straw. Bioresource Technology. 94:223 - 228.

CORREDOR, J. 2006. El residuo líquido de las curtiembres estudio de caso: cuenca alta del Río Bogotá. Ciencia e Ingeniería Neogranadina. 16(2):14 - 28.

COSTA, P.; CORRAL, R.; ILLERA, M.; LÓPEZ, E. 2010. Empleo de un compost de algas y restos de pescado como sustrato para la producción de plantas hortícolas. Recursos Rurais 6:89 - 94.

CUERVO, N. 2010. Estudio del proceso de compostaje de los lodos producidos en la operación de pelambre en la industria del curtido de pieles. Universidad Nacional, Bogotá, Colombia. 14p.

DAMA. DEPARTAMENTO TÉCNICO ADMINISTRATIVO DEL MEDIO AMBIENTE. 2004. Guía ambiental para el sector curtiembres. En http: // www.cortolima.gov. co /SIGAM/Series/curtiembres.pdf; consulta: febrero, 2016

GIL, M.; RUEDA, P.; SALGADO, A.; VARELA, A. 2006. Guía de uso de la tecnología EM en ganadería. FUNDASES. Colombia. 5 p.

GUZMÁN, K.; LUJÁN, M. 2010. Reducción de emisiones de la etapa de pelambre en el proceso de curtido de pieles. Rev. Acta Nova. 4(4):464 - 492.

HERRERA, C.; SAMPER, J.; CHARRY, A. 2015. Oportunidades de negocio en sectoriales y grupos poblaciones clave. Editorial: Corporación para el desarrollo de las Microempresas. Colombia. $124 \mathrm{p}$.

IBM. International Business Machine. 2013. SPSS (Statistical Package for Social Sciences). En: http://www03.ibm.com/press/us/en/background.wss; consulta: noviembre, 2015.

IGAC. 2006. Métodos analíticos de laboratorio de suelos. Ed. Instituto Geográfico Agustín Codazzi. Colombia. 648 p. 
INN. INSTITUTO NACIONAL DE NORMALIZACIÓN. 2005. Residuos sólidos. Norma Chilena 2880 "Compost - Clasificación y requisitos". En: http://www.sinia. cl/1292/; consulta: enero, 2016.

LABRADOR, J. 2001. La materia orgánica en los agroecosistemas. Ed. Ediciones MundiPrensa (España). 168 p.

LÓPEZ, W. 2010. Estudio del uso de residuos industriales no peligrosos a través del proceso de compostaje y su aplicación para el cultivo de maíz y frijol. En: http://www.repositoriodigital.ipn.mx/bitstream/handle/123456789/6940/TESIS; consulta: julio, 2016.

NAIR, J.; OKAMITSU, K. 2010. Microbial inoculants for small scale composting of putrescible kitchen wastes. Waste Manag. 30(6):977 - 982.

OSORIO, L.; MAARTEN, S.; SÁNZ, M.; SANTOS, T.; TOBÓN, C.; BELLO, T.; ESCAMILLA, C. 2011a. Iniciativas ambientales en la cuenca del Río Bogotá: Curtiembres de Villapinzón y Chocontá y Cuenca del Embalse Tominé. Primera edición. Opciones gráficas editores Ltda. Colombia. $644 \mathrm{p}$.

OSORIO, L.; SANTOS, T.; SÁNZ, M.; ARAGÓN, C.; TOBÓN, C.; BELLO, T.; ESCAMILLA, C. 2011b. La gestión ambiental y la inclusión social, condiciones para lograr la competitividad en micro y pequeñas industrias (MyPI). Primera edición. Opciones gráficas editores Ltda. Colombia. 160 p.

PALMERO, R. 2010. Elaboración de compost con restos vegetales por el sistema tradicional en pilas o montones. Servicio Técnico de Agricultura y Desarrollo Rural del Cabildo Insular de Tenerife. p6. En: http://www. ecohabitar.org/wp-content/uploads/2013/

10/elaboración-de-compost-con-restos-vegetales-1. pdf.; consulta: abril, 2016.

REYES, C.; LOBO, M.; FEHER, S. 2009. Informe Aspectos Ambientales, Legales y Socioeconómicos de las curtiembres. Secretaría de Industria, Comercio y PyME. En: http://myslide.es/documents/informe-aspectosambientales-legales-y-socioeconomicos-curtiembres-2009.html; consulta: septiembre, 2016.

RODRÍGUEZ, M.; CÓRDOVA, A. 2006. Manual de Compostaje Municipal, Tratamiento de Residuos Sólidos Urbanos. Programa Gestión Ambiental y Manejo Sustentable de los Recursos Naturales. Componente Gestión de Residuos Sólidos y Sitios Contaminados. Primera Edición. México. 22 p
ROMÁN, P.; MARTÍNEZ, M.; PANTOJA, A. 2013. Manual de Compostaje del Agricultor. Experiencias en América Latina. En: http://www.fao.org/3/a-i3388s.pdf; consulta: junio, 2016.

SAEZ, A. 2000. Optimización de los métodos para mejorar la calidad del compost de la fracción orgánica de los residuos sólidos urbanos. En: http://oa.upm.es/613/; consulta: febrero, 2016.

SÁNCHEZ, M.; ROIG, A.; PAREDES, C.; BERNAL, M. 2001. Nitrogen transformation during organic waste composting by the Rutgers system and its effects on $\mathrm{pH}, \mathrm{EC}$ and maturity of the composting mixtures. Bioresource Technology. 78(3):301 - 308.

SYLVIA, D.; HARTEL, P.; FUHRMANN, J.; ZUBERER, D. 2005. Principles and applications of soil microbiology. Ed. Prentice Hall. New Jersey. 640 p.

SWITCH, P. 2011. El manejo sostenible del agua en las ciudades del mañana. Convenio Universidad Nacional de Colombia, UNESCO- IHE de Holanda. 53 p.

SZTERN, D.; PRAVIA, M. 1999. Manual para la elaboración de compost, bases conceptuales y procedimiento. Organización Panamericana de la salud. En http://www. bvsops.org.uy/pdf/compost.pdf; consulta: mayo, 2016.

TORTAROLO, M.; PEREDA, M.; PALMA, M.; ARRIGO, N. 2008. Influence of inoculation of microorganisms on the temperature in the process of composting. Ci. Suelo (argentina). 26(1):41 - 50.

USEPA- AGENCIA DE PROTECCIÓN AMBIENTAL DE ESTADOS UNIDOS. 1995. A guide to the biosolids risk assessments for the EPA Part 503 Rule EPA/B32B-93-005. United States Environmental Protection Agency Office of Wastewater Management. Washinton, DC. $158 \mathrm{p}$.

VÁSQUEZ, M.; PRADA, P.; MONDRAGÓN, M. 2010. Optimización del proceso de compostaje de productos postcosecha (cereza) del café con la aplicación de microorganismos nativos. Cienc. Bioméd. 8(14):213 - 219.

VITERI, P.; CASTILLO, D.; VITERI, S. 2015. Desarrollo y evaluación de un inóculo de bacterias celulolíticas. Rev. U.D.C.A Act. \& Div. Cient. 18(1):207 - 215. 